\title{
D Upper Normal Limit of Serum Alanine Aminotransferase and Its Association with Metabolic Risk Factors in Pars Cohort Study
}

\author{
Faeze Sehatpour ${ }^{1}$, Alireza Salehi ${ }^{2, *}$, Hossein Molavi Vardanjani ${ }^{1}$, Hossein Poustchi ${ }^{3}$, Abdullah Gandomkar ${ }^{4}$, \\ Reza Malekzadeh ${ }^{3,4}$
}

1. MPH Department, Shiraz Medical School, Shiraz University of Medical Sciences, Shiraz, Iran

2. Research Center for Traditional Medicine and History of Medicine, Shiraz University of Medical Sciences, Shiraz, Iran

3. Liver, Pancreatic, and Biliary Diseases Research Center, Digestive Disease Research Institute, Tehran University of Medical Sciences, Tehran, Iran

4. Non-Communicable Disease Research Center, Shiraz University of Medical Sciences, Shiraz, Iran

\footnotetext{
* Corresponding Author:

Alireza Salehi, MD, MPH, PhD of Epidemiology

Research Center for Traditional Medicine and History of Medicine, Shiraz University of Medical Sciences, Shiraz, Iran Tel: + 987132337589

Fax: + 987132338476

Email: salehialireza45@yahoo.com
}

Received: 19 Jun. 2019

Accepted: 18 Nov. 2019

\section{ABSTRACT}

\section{BACKGROUND}

The range of serum alanine aminotransferase (ALT) varies in different sub-populations or countries. Its population-specific cut-off points may provide a more effective screening tool for non-alcoholic fatty liver disease (NAFLD).

Objectives

To investigate the upper normal level (UNL) of ALT and its association with metabolic syndrome (MS) in a semi-urban population in southern Iran.

\section{METHODS}

The baseline data of Pars Cohort Study was used. A total of 9264 subjects aged 40-75 years were enrolled. UNL of ALT was estimated based on 95 percentile of ALT in participants who had body mass index $(\mathrm{BMI})<25$. Multivariable logistic regression was applied and adjusted odds ratio (OR) and its $95 \%$ confidence interval (CI) were estimated.

\section{RESULTS}

95 percentile of ALT was $41.71 \mathrm{U} / \mathrm{L}$ and $32.9 \mathrm{U} / \mathrm{L}$ in men and women, respectively. Abnormal waist circumference (OR: 1.72, 95\%CI: 1.34, 2.21), triglyceride (OR: $1.63,95 \% \mathrm{CI}: 1.25,2.13$ ), fasting blood sugar (OR: 1.69, 95\%CI: 1.32, 2.16), cholesterol level (OR: 1.06, 95\%CI: 1.03, 1.09) and systolic blood pressure (OR: 1.08, 95\%CI: 1.01, 1.16) were independently associated with ALT.

\section{CONCLUSION}

UNL of ALT in southern Iranian women is lower than the current recommended level, while these are almost the same for men. MS components are highly common in southern Iran and are associated with elevated serum ALT. Further studies are recommended to estimate the UNL of serum ALT among the Iranian population with NAFLD.

\section{KEYWORDS:}

Alanine aminotransferase, Metabolic syndrome, Upper normal limit, Pars Cohort Study, Iran

\section{Please cite this paper as:}

Sehatpour F, Salehi A, Molavi Vardanjani H, Poustchi H, Gandomkar A, Malekzadeh R. Upper Normal Limit of Serum Alanine Aminotransferase and Its Association with Metabolic Risk Factors in Pars Cohort Study. Middle East J Dig Dis 2020;12:19-26. doi: 10.15171/mejdd.2020.159.

\section{INTRODUCTION}

Serum alanine aminotransferase (ALT), as a hepatic-predictive value for chronic metabolic diseases, is an important factor for screening non-alcoholic fatty liver disease (NAFLD). ${ }^{1,2}$ However, its range varies in different populations 
based on age, sex, ethnicity, body mass index (BMI), the prevalence of NAFLD, serum levels of low-density lipoprotein (LDL), and components of metabolic syndrome (MS). ${ }^{3-6}$ Therefore, population-specific cut-offs for ALT may provide a more effective screening tool for NAFLD in each population. ${ }^{7.8}$

The current UNL of serum ALT for the screening of liver diseases is $40 \mathrm{U} / \mathrm{L}$ (ranging from 30-50). However, this is not very accurate for the diagnosis of NAFLD, and considering this range of ALT for all populations may lead to misdiagnosis of some subjects. ${ }^{9-11}$

There are controversies on the cut-off point of ALT in Iranian adults and the correlation of available cut-offs with NAFLD, MS, or other chronic diseases. .,12,13 $^{2}$

We analyzed the baseline data of the participants of Pars Cohort Study to investigate the normal range of ALT and its association with MS in a semi-urban population in southern Iran.

\section{MATERIALS AND METHODS}

\section{Study subjects and setting:}

The baseline data of Pars Cohort Study was used. In brief, in the PCS, inhabitants of Valashahr city, with an age range of 40-75 years (totally 9721) were invited to participate in the study. They were introduced to the aims and phases of PCS. The participants who gave informed consent were interviewed and underwent physical examinations and blood sampling. A total of 9264 subjects (95\%) participated in the PCS. Both ethical review committees of Shiraz University of Medical Sciences (SUMS) and Digestive Disease Research Institute of Tehran University of Medical Sciences (DDRI) approved the PCS protocol. All participants completed and signed informed consent before their participation in the PCS. Further details are presented in the PCS profile. ${ }^{14}$

\section{Exclusion criteria:}

PCS participants who had a history of diagnosed liver disease, renal failure, cancer, ischemic heart disease, stroke, chronic hemolysis, acute infection, viral hepatitis (subjects who had positive viral markers), those who took hepatotoxic drugs, ${ }^{15,16}$ as well as alcohol drinkers (with an intake of more than 20 grams alcohol per day) were excluded from the study.

\section{Variables measurement:}

More than 200 socio-demographic, medical history, and biochemical and biomedical variables were collected in the PCS at the baseline. ${ }^{14}$ Data on demographic characteristics (age, sex, ethnicity, weight, height, body waist circumference (WC), blood pressure (BP), history of physical activity, medication history, history of chronic diseases, alcohol consumption (the number and type of drinks per day), cigarette smoking, opium usage, fasting blood sugar (FBS), lipid profile, and ALT level were selected for this study.

\section{Data collection:}

Biochemical parameters including the serum ALT were measured by using the auto-analyzer, model Bt1500, and the kits were made by Parse Azmoon Company.

We defined MS based on the definition of the We defined MS based on the definition of the Azizi et al (as follows: any three of the following five factors: WC $\geq 90 \mathrm{~cm}$, triglyceride (TG) $\geq 150 \mathrm{mg} / \mathrm{dL}, \mathrm{FBS} \geq 100 \mathrm{mg} /$ $\mathrm{dL}$, or previously diagnosed type $2 \mathrm{DM}$, high-density lipoprotein (HDL) $<40 \mathrm{mg} / \mathrm{dL}$ in men, and $<50 \mathrm{mg} / \mathrm{dL}$ in women, systolic blood pressure (SBP) $\geq 130 \mathrm{~mm} \mathrm{Hg}$ or diastolic blood pressure (DBP) $\geq 85 \mathrm{~mm} \mathrm{Hg}$, or treatment of previously diagnosed HTN $).{ }^{17}$

Body mass index (BMI) was categorized into three groups $\left(<25 \mathrm{~kg} / \mathrm{m}^{2}, \geq 25-30<\mathrm{kg} / \mathrm{m}^{2}\right.$ and $\left.\geq 30 \mathrm{~kg} / \mathrm{m}^{2}\right)$. Also, age was categorized into four groups (40-49, 50$59,60-69$, and $\geq 70$ years). By applying a recommended method for analysis of the International Physical Activity Questionnaire (IPAQ), the value of Metabolic Equivalent of Task (MET) scores was calculated as a measure of physical activity and then categorized into its tertiles (low, medium, and high physical activity). To estimate the prevalence of abnormal metabolic factors in association with the serum ALT level, we defined four categories for the ALT level $(<$ mean, mean to mean $+\mathrm{SD}$, mean $+\mathrm{SD}$ to mean $+2 \mathrm{SD}$ and $\geq$ mean $+2 \mathrm{SD}$ ) in men and women separately.

\section{Statistical Analysis:}

We estimated the proportions and means \pm standard deviations (SD) to describe the data. $95 \%$ confidence interval of prevalence was estimated. Two-sided independent sample $t$ test and Chi-square test were used. UNL of ALT 
was estimated based on 95 percentage of ALT distribution in participants who had BMI $<25 \mathrm{~kg} / \mathrm{m}^{2}$. The effect of confounding variables was controlled using multivariable logistic regression. Two logistic regression models were fitted to assess the correlates of "current UNL of serum ALT" (what was identified in this study) and "past UNL of serum ALT" (ALT > 40). The significance level was set at 0.05. Stata software version11.2 (StataCorp. LP) was used for data analysis.

\section{RESULTS}

6459 out of 9264 subjects were included in this study. Female/male ratio was 1:1.01 (3217/3242). MS was significantly more prevalent in women than men $(\mathrm{n}=862$; prevalence $=26.9 \%, 95 \% \mathrm{CI}: 25.36-28.46$ vs. $\mathrm{n}=542$; prevalence $=16.7 \%, 95 \% \mathrm{CI}: 15.42-18.03 ; p<0.001)$. For $1.1 \%(\mathrm{n}=73)$ of participants, including: $1.6 \%(\mathrm{n}$ $=52)$ of female participants and $0.6 \%(n=21)$ of male ones, all of the MS components were abnormal (table 1).

Mean ALT was significantly higher in men than women (23.78 \pm 15.08 vs. $18.65 \pm 12.24 ; p<0.001)$. In participants with normal BMI, 95 percentile of ALT was $41.71 \mathrm{U} / \mathrm{L}$ and $32.9 \mathrm{U} / \mathrm{L}$ in men and women, respectively (table2). In women with a history of DM, the mean of ALT was significantly higher than non-diabetics $(22.07 \pm 14.97$ vs. $18.32 \pm 11.88 ; p<0.001)$, but there was no statistically significant difference between diabetic and non-diabetic men $(24.88 \pm 17.34$ vs. $23.73 \pm 14.97 ; p=0.364)$.

Abnormal WC was found to be a risk factor for increasing the serum ALT in both current UNL and in ALT $>40 \mathrm{U} / \mathrm{L}$ (OR: 1.72, 95\%CI: 1.34, 2.21 vs. OR: $1.42,95 \% \mathrm{CI}: 1.09$, 1.86), according to multivariable analyses (table 3 ).

The prevalence of high BP increased due to the increase in the serum ALT in men but not in women (table 4).

\section{DISCUSSION}

The UNL of serum ALT level is about $40 \mathrm{U} / \mathrm{L}$ in laboratory references, but according to different studies in different regions, the cut-off point of serum ALT needs to be revised. ${ }^{1,8,18}$ In the current study, among the inhabitants in southern Iran, in those with normal BMI, 95 percentile of serum ALT was 41.71 and 32.9 in male and female subjects, respectively. The upper limit of the serum ALT level was significantly higher in subjects who had at least one abnormality in their metabolic syndrome components.
In our previous study in northern Iran, UNL of the serum ALT was 37.5 in men and 36 in women, which were different in comparison with our current estimates. This difference may be due to the difference in our sampling population, one from the north and another from the south of Iran. ${ }^{12}$

Our estimation for UNL is considerably higher than some previous reports..$^{4,7,12,18}$ It may be due to the difference in the studied population or methodological considerations such as statistical methods applied for the estimation of UNL and inclusion of participants with NAFLD. Lack of any information about participants with NAFLD was another point that must be considered. ${ }^{2,11}$ In addition, differences between laboratories and various detection kits of ALT may cause test heterogeneity in different studies and regions. ${ }^{10}$

All MS components were positively correlated with an increase in the serum ALT. These findings were consistent with those of the previous studies showing that higher levels of serum ALT were associated with MS components and the development of MS. ${ }^{19,20}$ In 2012 in China, the cut-off point for the serum ALT level, which causes metabolic disorder affecting the liver, was 21-25 IU/L for men and 17-22 IU/L for women. ${ }^{8}$ In recent studies, NAFLD has been a prerequisite for the development of metabolic diseases. ${ }^{21}$ By an increase in the level of liver enzyme, the risk of the presence of NAFLD will increase, even in normal population. It seems to be necessary to investigate people whose ALT level is even in the upper normal range. ${ }^{8}$

Overall, in subjects who have high physical activity in our study, UNL of ALT was lower in comparison to people with low and moderate activity. Previous reports have shown a significant decrease in the liver enzyme by an aerobic exercise. ${ }^{22-24}$ These results may be influenced by the effect of exercise on MS components, especially in the distribution of visceral fat and insulin resistance that affects the serum ALT. $^{25}$ More studies are recommended to evaluate the direct effect of physical activity on serum ALT. 22,24,26

According to multivariable analysis, age had a negative correlation with elevated serum ALT. This finding was in the same line with that of the study published by Dong and his collaborators in 2011. In Dong's study, by analyzing two cohorts of individuals who participated in the Rancho Bernardo's study, with an increase in age, the 
Table 1: The participants' characteristics

\begin{tabular}{|c|c|c|c|c|}
\hline Variables & $\begin{array}{c}\text { Total }=6459 \\
\text { n(Prevalence) } \\
95 \% \text { CI }\end{array}$ & $\begin{array}{c}\text { Male }=3242 \\
\text { n(Prevalence) } \\
95 \% \text { CI }\end{array}$ & $\begin{array}{c}\text { Female }=3217 \\
\text { n(Prevalence) } \\
95 \% \text { CI }\end{array}$ & $p$ value \\
\hline $\begin{array}{l}\text { Age } \\
(\text { mean } \pm \mathrm{SD} \text { in years) }\end{array}$ & $51.66 \pm 9.25$ & $51.73 \pm 9.5$ & $51.6 \pm 9.0$ & 0.59 \\
\hline $\begin{array}{l}\text { BMI } \\
\left(\text { mean } \pm \mathrm{SD}, \mathrm{kg} / \mathrm{m}^{2}\right)\end{array}$ & $25.54 \pm 4.63$ & $24.21 \pm 4.04$ & $26.88 \pm 4.8$ & $<0.001$ \\
\hline $\mathrm{BMI}<25 \mathrm{~kg} / \mathrm{m}^{2}$ & $\begin{array}{c}3035(47.2) \\
46.0,48.4\end{array}$ & $\begin{array}{l}1901(58.9) \\
57.2,60.6\end{array}$ & $\begin{array}{l}1134(35.4) \\
33.7,37.1\end{array}$ & $<0.001$ \\
\hline $25 \leq \mathrm{BMI}<30 \mathrm{~kg} / \mathrm{m}^{2}$ & $\begin{array}{c}2344(36.5) \\
35.3,37.7\end{array}$ & $\begin{array}{l}1055(32.7) \\
31.1,34.3\end{array}$ & $\begin{array}{l}1289(40.3) \\
38.5,42.0\end{array}$ & $<0.001$ \\
\hline $\mathrm{BMI} \geq 30 \mathrm{~kg} / \mathrm{m}^{2}$ & $\begin{array}{c}1049(16.3) \\
15.4,17.2\end{array}$ & $\begin{array}{l}271(8.4) \\
8.3,8.5\end{array}$ & $\begin{array}{l}778(24.3) \\
22.8,25.8\end{array}$ & $<0.001$ \\
\hline $\mathrm{WC} \geq 95 \mathrm{~cm}$ & $\begin{array}{c}2331(36.3) \\
35.13,37.49\end{array}$ & $\begin{array}{c}946(29.3) \\
27.74,30.9\end{array}$ & $\begin{array}{c}1385(43.3) \\
41.58,45.03\end{array}$ & $<0.001$ \\
\hline $\mathrm{FBS} \geq 100 \mathrm{mg} / \mathrm{dL}$ & $\begin{array}{l}2455(38) \\
36.8,39.2\end{array}$ & $\begin{array}{l}1186(36.6) \\
34.9,38.3\end{array}$ & $\begin{array}{l}1269(39.4) \\
37.7,41.1\end{array}$ & 0.018 \\
\hline Low HDL H.* $^{* *}$ & $\begin{array}{l}886(13.7) \\
12.9,14.6\end{array}$ & $\begin{array}{c}256(7.9) \\
7.7,8.0\end{array}$ & $\begin{array}{l}630(19.6) \\
18.2,21.0\end{array}$ & $<0.001$ \\
\hline $\mathrm{TG} \geq 150 \mathrm{mg} / \mathrm{dL}$ & $\begin{array}{c}2430(37.6) \\
36.4,38.8\end{array}$ & $\begin{array}{l}1179(36.4) \\
34.7,38.1\end{array}$ & $\begin{array}{l}1251(38.9) \\
37.2,40.6\end{array}$ & 0.037 \\
\hline High $\mathrm{BP}^{* * * *}$ & $\begin{array}{c}1315(20.4) \\
19.4,21.4\end{array}$ & $\begin{array}{l}617(19.0) \\
17.7,20.4\end{array}$ & $\begin{array}{l}698(21.7) \\
20.3,23.1\end{array}$ & 0.008 \\
\hline MS & $\begin{array}{l}1404(21.8) \\
20.8,22.83\end{array}$ & $\begin{array}{c}542(16.7) \\
15.42,18.03\end{array}$ & $\begin{array}{c}862(26.9) \\
25.36,28.46\end{array}$ & $<0.001$ \\
\hline History of HTN & $\begin{array}{c}643(10) \\
9.3,10.7\end{array}$ & $\begin{array}{l}176(5.4) \\
5.2,5.6\end{array}$ & $\begin{array}{l}467(14.5) \\
13.3,15.7\end{array}$ & $<0.001$ \\
\hline History of $\mathrm{T}^{2} \mathrm{DM}$ & $\begin{array}{c}438(6.8) \\
6.7,6.9\end{array}$ & $\begin{array}{l}150(4.6) \\
4.4,4.8\end{array}$ & $\begin{array}{l}288(9.0) \\
8.9,9.1\end{array}$ & $<0.001$ \\
\hline History of opium use & $\begin{array}{l}514(8) \\
7.9,8.1\end{array}$ & $\begin{array}{l}496(15.3) \\
14.1,16.6\end{array}$ & $\begin{array}{c}18(0.6) \\
0.58,0.62\end{array}$ & $<0.001$ \\
\hline Current cigarette smoking & $\begin{array}{l}977(15.2) \\
14.3,16.1\end{array}$ & $\begin{array}{l}959(29.6) \\
28.0,31.2\end{array}$ & $\begin{array}{c}18(0.6) \\
0.58,0.62\end{array}$ & $<0.001$ \\
\hline \multicolumn{5}{|l|}{ Physical activity } \\
\hline Low & $\begin{array}{c}1805(28) \\
26.9,29.1\end{array}$ & $\begin{array}{l}666(20.6) \\
19.2,22.0\end{array}$ & $\begin{array}{l}1139(35.4) \\
33.7,37.1\end{array}$ & $<0.001$ \\
\hline Moderate & $\begin{array}{c}2203(34.1) \\
32.9,35.2\end{array}$ & $\begin{array}{l}942(29.1) \\
27.5,30.7\end{array}$ & $\begin{array}{c}1261(39.2) \\
37.5,40.9\end{array}$ & $<0.001$ \\
\hline High & $\begin{array}{c}2445(37.9) \\
36.7,9.1\end{array}$ & $\begin{array}{c}1631(50.4) \\
48.7,52.1\end{array}$ & $\begin{array}{l}814(25.3) \\
23.8,26.8\end{array}$ & $<0.001$ \\
\hline
\end{tabular}

n, number of participants; BMI, body mass index; WC, waist circumference; FBS, fasting blood sugar; HDL, high-density lipoprotein; TG, triglyceride; BP, blood pressure; MS, metabolic syndrome; HTN, hypertension; T2DM, diabetes mellitus type 2

"HDL $<40$ in men, HDL $<50 \mathrm{mg} / \mathrm{dL}$ in women

${ }^{*+*}$ Systolic blood pressure $\geq 130$ and/ or diastolic blood pressure $\geq 85 \mathrm{~mm} \mathrm{Hg}$

serum level of ALT decreased, especially in old ages. ${ }^{27}$ By an increase in age, the function of the liver decreases and it may cause a decrease in the level of liver enzymes. ${ }^{28}$ It seems to be necessary to define the UNL of serum ALT in different age groups in laboratory reference values.

Although this is a cross-sectional study on 40-75 years old adults of a semi-rural population, one of the most important strengths of the current study was its adequacy of the sample size which enabled us to do an in-depth stratified analysis. We also used baseline data of the Pars Cohort Study, which has been collected in a valid and standardized way.

Lack of any data about the subjects who were diagnosed with NAFLD (by sonography or liver biopsy) was an important limitation in our study and further investigation is required to determine an exact cut-off point for the 
Table 2: ALT features in different subgroups

\begin{tabular}{|c|c|c|c|c|c|c|c|c|}
\hline ALT U/L & Sex & Subgroups & N (\%) & $\begin{array}{c}\text { Median } \\
\text { U/L }\end{array}$ & MIN, MAX & Mean \pm SD & $\mathbf{9 5}^{\text {th }}$ & $p$ value \\
\hline \multirow{2}{*}{$\begin{array}{l}\text { ALT U/L } \\
\text { overall }\end{array}$} & $\mathrm{M}$ & & 3242 & 19.94 & $1.81,137.86$ & $23.78 \pm 15.08$ & 51.68 & \multirow{2}{*}{$<0.001$} \\
\hline & $\mathrm{F}$ & & 3217 & 15.65 & $1.1,153.64$ & $18.65 \pm 12.24$ & 37.94 & \\
\hline \multirow{6}{*}{ BMI } & \multirow{3}{*}{ M } & $<25 \mathrm{~kg} / \mathrm{m}^{2}$ & $\begin{array}{c}1901 \\
(58.9)\end{array}$ & 17.05 & $1.81,137.86$ & $20.34 \pm 12.96$ & 41.71 & \multirow{3}{*}{$<0.001$} \\
\hline & & $\begin{array}{c}\geq 25-30< \\
\mathrm{kg} / \mathrm{m}^{2}\end{array}$ & $\begin{array}{c}1055 \\
(32.7)\end{array}$ & 24.03 & $5.62,126.77$ & $27.97 \pm 16.13$ & 57.29 & \\
\hline & & $\geq 30 \mathrm{~kg} / \mathrm{m}^{2}$ & $271(8.4)$ & 26.1 & $7.47,107.33$ & $31.7 \pm 17.53$ & 66.74 & \\
\hline & \multirow{3}{*}{$\mathrm{F}$} & $<25 \mathrm{~kg} / \mathrm{m}^{2}$ & $\begin{array}{c}1134 \\
(35.4)\end{array}$ & 14.03 & $1.1,147.4$ & $16.34 \pm 10.49$ & 32.9 & \multirow{3}{*}{$<0.001$} \\
\hline & & $\begin{array}{c}\geq 25-30< \\
\mathrm{kg} / \mathrm{m}^{2}\end{array}$ & $\begin{array}{c}1289 \\
(40.3)\end{array}$ & 16.41 & $1.81,153.64$ & $19.53 \pm 13.0$ & 40.01 & \\
\hline & & $\geq 30 \mathrm{~kg} / \mathrm{m}^{2}$ & $\begin{array}{c}778 \\
(24.3)\end{array}$ & 16.91 & $1.31,124.4$ & $20.63 \pm 12.86$ & 41.41 & \\
\hline \multirow{4}{*}{ TG } & \multirow{2}{*}{ M } & $\geq 150 \mathrm{mg} / \mathrm{dL}$ & $\begin{array}{c}1179 \\
(36.4)\end{array}$ & 24.02 & $4.25,135.8$ & $28.21 \pm 16.77$ & 61.55 & \multirow{2}{*}{$<0.001$} \\
\hline & & $<150 \mathrm{mg} / \mathrm{dL}$ & $\begin{array}{l}2063 \\
(63.6)\end{array}$ & 18.0 & $1.81,137.86$ & $21.26 \pm 13.39$ & 44.21 & \\
\hline & \multirow{2}{*}{$\mathrm{F}$} & $\geq 150 \mathrm{mg} / \mathrm{dL}$ & $\begin{array}{c}1251 \\
(38.9) \\
\end{array}$ & 17.37 & $1.5,151.46$ & $20.65 \pm 12.38$ & 43.04 & \multirow{2}{*}{$<0.00$} \\
\hline & & $<150 \mathrm{mg} / \mathrm{dL}$ & $\begin{array}{c}1966 \\
(61.1)\end{array}$ & 14.76 & $1.1,153.64$ & $17.39 \pm 11.98$ & 34.33 & \\
\hline \multirow{4}{*}{ FBS } & \multirow{2}{*}{ M } & $\geq 100 \mathrm{mg} / \mathrm{dL}$ & $\begin{array}{c}1186 \\
(36.6)\end{array}$ & 21.34 & $2.7,125.05$ & $25.49 \pm 16.26$ & 56.32 & \multirow{2}{*}{$<0.001$} \\
\hline & & $<100 \mathrm{mg} / \mathrm{dL}$ & $\begin{array}{l}2056 \\
(63.4)\end{array}$ & 19.09 & $1.81,137.86$ & $22.8 \pm 14.27$ & 47.83 & \\
\hline & \multirow{2}{*}{$\mathrm{F}$} & $\geq 100 \mathrm{mg} / \mathrm{dL}$ & $\begin{array}{c}1269 \\
(39.4)\end{array}$ & 16.98 & $1.5,151.46$ & $20.3 \pm 12.82$ & 42.58 & \multirow{2}{*}{$<0.00$} \\
\hline & & $<100 \mathrm{mg} / \mathrm{dL}$ & $\begin{array}{c}1948 \\
(60.6)\end{array}$ & 14.82 & $1.1,153.64$ & $17.58 \pm 11.72$ & 35.35 & \\
\hline \multirow{4}{*}{ HDL } & \multirow[b]{2}{*}{ M } & $<40 \mathrm{mg} / \mathrm{dL}$ & $256(7.9)$ & 23.05 & $2.7,125.05$ & $27.27 \pm 17.69$ & 62.25 & \multirow[b]{2}{*}{0.001} \\
\hline & & $\geq 40 \mathrm{mg} / \mathrm{dL}$ & $\begin{array}{l}2986 \\
(92.1)\end{array}$ & 19.72 & $1.81,137.86$ & $23.49 \pm 14.8$ & 50.73 & \\
\hline & \multirow{2}{*}{$\mathrm{F}$} & $<50 \mathrm{mg} / \mathrm{dL}$ & $\begin{array}{c}630 \\
(19.6)\end{array}$ & 16.26 & $4.32,147.41$ & $20.1 \pm 14.33$ & 43.31 & \multirow{2}{*}{0.004} \\
\hline & & $\geq 50 \mathrm{mg} / \mathrm{dL}$ & $\begin{array}{c}2587 \\
(80.4)\end{array}$ & 15.52 & $1.1,153.64$ & $18.3 \pm 11.64$ & 36.7 & \\
\hline \multirow{4}{*}{$\begin{array}{l}\mathrm{High}^{*} \\
\text { BP }\end{array}$} & \multirow{2}{*}{$\mathrm{M}$} & High & $\begin{array}{c}617 \\
(19.0)\end{array}$ & 21.56 & $3.32,135.8$ & $25.61 \pm 16.38$ & 54.94 & \multirow{2}{*}{0.001} \\
\hline & & Normal & $\begin{array}{c}2622 \\
(81.0) \\
\end{array}$ & 19.57 & $1.81,137.86$ & $23.36 \pm 14.74$ & 50.86 & \\
\hline & \multirow{2}{*}{ F } & High & $\begin{array}{c}698 \\
(21.7)\end{array}$ & 16.01 & $1.31,116.28$ & $18.73 \pm 10.98$ & 38.94 & \multirow{2}{*}{0.833} \\
\hline & & Normal & $\begin{array}{l}2516 \\
(78.3)\end{array}$ & 15.57 & $1.1,153.64$ & $18.62 \pm 12.56$ & 37.75 & \\
\hline \multirow{4}{*}{ WC } & \multirow{2}{*}{$\mathrm{M}$} & $\geq 95 \mathrm{~cm}$ & $\begin{array}{c}946 \\
(29.3)\end{array}$ & 24.79 & $7.21,126.77$ & $24.79 \pm 16.85$ & 64.81 & \multirow{2}{*}{$<0.001$} \\
\hline & & $<95 \mathrm{~cm}$ & $\begin{array}{l}2281 \\
(70.7)\end{array}$ & 18.09 & $1.81,137.86$ & $21.55 \pm 13.5$ & 45.5 & \\
\hline & F & $\geq 95 \mathrm{~cm}$ & $\begin{array}{c}1385 \\
(43.3)\end{array}$ & 17.06 & $1.31,153.64$ & $20.62 \pm 13.45$ & 41.38 & $<0001$ \\
\hline & $\Gamma^{2}$ & $<95 \mathrm{~cm}$ & $\begin{array}{c}1816 \\
(56.7)\end{array}$ & 14.69 & 1. $1,151.46$ & $17.18 \pm 11.04$ & 34.92 & -0.001 \\
\hline
\end{tabular}




\begin{tabular}{|c|c|c|c|c|c|c|c|c|}
\hline ALT U/L & Sex & $\begin{array}{c}\text { Sub- } \\
\text { groups }\end{array}$ & N (\%) & $\underset{\text { U/L }}{\text { Median }}$ & MIN, MAX & Mean \pm SD & $95^{\text {th }}$ & $p$ value \\
\hline \multirow{4}{*}{ MS } & \multirow{2}{*}{$\mathrm{M}$} & Yes & $\begin{array}{c}542 \\
(16.7) \\
\end{array}$ & 25.1 & $8.13,125.05$ & $30.61 \pm 18.5$ & 67.09 & \multirow{2}{*}{$<0.001$} \\
\hline & & No & $\begin{array}{c}2629 \\
(83.3)\end{array}$ & 18.73 & $1.81,137.86$ & $22.41 \pm 13.91$ & 46.68 & \\
\hline & \multirow{2}{*}{$\mathrm{F}$} & Yes & $\begin{array}{c}862 \\
(26.9) \\
\end{array}$ & 17.83 & $1.81,120.2$ & $21.3 \pm 12.59$ & 44.55 & \multirow{2}{*}{$<0.001$} \\
\hline & & No & $\begin{array}{c}2345 \\
(71.4)\end{array}$ & 14.96 & $1.1,153.64$ & $17.69 \pm 11.98$ & 35.61 & \\
\hline \multirow{6}{*}{$\begin{array}{l}\text { Physical } \\
\text { Activity }\end{array}$} & \multirow{3}{*}{$\mathrm{M}$} & Low & $\begin{array}{c}666 \\
(20.6)\end{array}$ & 20.36 & $2.7,135.2$ & $25.01 \pm 17.39$ & 56.87 & \multirow{3}{*}{$<0.001^{* *}$} \\
\hline & & Moderate & $\begin{array}{c}942 \\
(29.1)\end{array}$ & 20.69 & $1.81,135.8$ & $24.99 \pm 16.2$ & 54.73 & \\
\hline & & High & $\begin{array}{c}1631 \\
(50.4)\end{array}$ & 19.29 & $3.32,137.86$ & $22.59 \pm 13.2$ & 45.59 & \\
\hline & \multirow{3}{*}{$\mathrm{F}$} & Low & $\begin{array}{c}1139 \\
(35.4)\end{array}$ & 15.71 & $1.1,151.46$ & $18.27 \pm 11.17$ & 37.42 & \multirow{3}{*}{$0.491^{* *}$} \\
\hline & & Moderate & $\begin{array}{c}1261 \\
(39.2) \\
\end{array}$ & 15.68 & $1.31,153.64$ & $19.17 \pm 13.45$ & 38.9 & \\
\hline & & High & $\begin{array}{c}814 \\
(25.3)\end{array}$ & 15.49 & $1.7,147.41$ & $18.41 \pm 11.66$ & 37.28 & \\
\hline \multirow{8}{*}{ Age } & \multirow{4}{*}{ M } & $(40-49)$ & $\begin{array}{c}1597 \\
(49.3)\end{array}$ & 22.64 & $3.33,137.86$ & $27.01 \pm 16.75$ & 60.15 & \multirow{4}{*}{$<0.001^{* * *}$} \\
\hline & & $(50-59)$ & $\begin{array}{c}1017 \\
(31.4)\end{array}$ & 19.39 & $3.56,122.91$ & $21.74 \pm 12.23$ & 43.15 & \\
\hline & & $(60-69)$ & $\begin{array}{c}432 \\
(13.3)\end{array}$ & 17.27 & $3.25,135.8$ & $20.15 \pm 4.44$ & 35.89 & \\
\hline & & $\geq 70$ & $196(6)$ & 14.5 & $1.81,47.35$ & $16.11 \pm 7.07$ & 31.25 & \\
\hline & \multirow{4}{*}{$\mathrm{F}$} & $(40-49)$ & $\begin{array}{c}1593 \\
(49.5)\end{array}$ & 15.31 & $1.1,147.41$ & $18.35 \pm 12.34$ & 38.17 & \multirow{4}{*}{$<0.001^{* * *}$} \\
\hline & & $(50-59)$ & $\begin{array}{c}944 \\
(29.3) \\
\end{array}$ & 16.99 & $2.04,151.46$ & $19.83 \pm 12.1$ & 39.93 & \\
\hline & & $(60-69)$ & $\begin{array}{l}548 \\
(17)\end{array}$ & 15.67 & $1.81,153.64$ & $18.48 \pm 12.82$ & 36.02 & \\
\hline & & $\geq 70$ & $\begin{array}{l}132 \\
(4.1)\end{array}$ & 12.94 & $4.5,58.29$ & $14.73 \pm 7.63$ & 31.49 & \\
\hline
\end{tabular}

ALT, alanine aminotransferase; N, number of participants; M, male; F, female; BMI, body mass index; TG, triglyceride; FBS, fasting blood sugar; HDL, high-density lipoprotein; BP, blood pressure; WC, waist circumference; MS, metabolic syndrome.

"Systolic blood pressure $\geq 130$ and/or diastolic blood pressure $\geq 85 \mathrm{~mm} \mathrm{Hg}$

"Mean of high physical activity group compared with two other groups overal

"*"Mean of age $>70$ compared with three other age groups overall

Table 3: Serum ALT correlates in the Pars cohort study, southern Iran, 2014

\begin{tabular}{|c|c|c|c|c|}
\hline \multirow{2}{*}{ Variables } & \multicolumn{2}{|c|}{ Current UNL of serum ALT } & \multicolumn{2}{|c|}{ Past UNL of serum ALT } \\
\hline & Crude. OR & Adj. OR & Crude. OR & Adj. OR \\
\hline Age (year) & $0.95(0.94,0.96)$ & $0.94(0.92,0.95)$ & $0.94(0.93,0.95)$ & $0.93(0.92,0.95)$ \\
\hline $\mathrm{WC} \geq 95 \mathrm{~cm}$ & $2.29(1.8,2.92)$ & $1.72(1.34,2.21)$ & $2.12(1.75,2.57)$ & $1.42(1.09,1.86)$ \\
\hline $\mathrm{BMI}>25 \mathrm{~kg} / \mathrm{m}^{2}$ & - & - & $2.19(1.78,2.68)$ & $1.65(1.24,2.21)$ \\
\hline Cholesterol mg/dL & $1.08(1.05,1.1)$ & $1.06(1.03,1.09)$ & $1.06(1.04,1.09)$ & $1.07(1.04,1.09)$ \\
\hline Male sex & - & - & $2.54(2.07,3.13)$ & $3.39(2.71,4.22)$ \\
\hline $\mathrm{TG} \geq 150 \mathrm{mg} / \mathrm{dL}$ & $2.38(2.0,3.85)$ & $1.63(1.25,2.13)$ & $2.48(2.04,3.0)$ & $1.59(1.28,1.98)$ \\
\hline $\mathrm{FBS} \geq 100 \mathrm{mg} / \mathrm{dL}$ & $1.6(1.34,1.9)$ & $1.69(1.32,2.16)$ & $1.63(1.35,1.98)$ & $1.52(1.24,1.86)$ \\
\hline SBP mm Hg & $1.09(1.02,1.16)$ & $1.08(1.01,1.16)$ & $1.07(1.02,1.12)$ & $1.07(1.0,1.13)$ \\
\hline
\end{tabular}

aAbbreviations: adj, adjusted; OR, odds ratio; WC, waist circumference; BMI, body mass index; TG, triglyceride; FBS, fasting blood sugar; SBP; systolic blood pressure 
Table 4: Percentage of abnormality in metabolic syndrome components by serum ALT levels

\begin{tabular}{|c|c|c|c|c|c|c|}
\hline \multicolumn{7}{|c|}{ ALT,U/L } \\
\hline Variables & $\mathbf{N}$ & $<$ Mean & $\begin{array}{c}\text { Mean to } \\
\text { Mean + 1SD }\end{array}$ & $\begin{array}{c}\text { Mean + 1SD to } \\
\text { Mean + 2SD }\end{array}$ & $\geq$ Mean + 2SD & $p^{*}$ \\
\hline \multicolumn{7}{|c|}{$\underline{\text { Male }}$} \\
\hline Overall & 3242 & 2068 & 820 & 214 & 140 & - \\
\hline High BP mm Hg & 617 & 17.3 & 22.6 & 19.6 & 22.9 & 0.007 \\
\hline $\mathrm{FBS} \geq 100 \mathrm{mg} / \mathrm{dL}$ & 1186 & 33.9 & 39.9 & 42.5 & 47.9 & $<0.001$ \\
\hline $\mathrm{TG} \geq 150 \mathrm{mg} / \mathrm{dL}$ & 1179 & 27.9 & 48.8 & 55.1 & 60.0 & $<0.001$ \\
\hline $\mathrm{HDL}<40 \mathrm{mg} / \mathrm{dL}$ & 256 & 6.5 & 10.2 & 9.3 & 12.9 & 0.001 \\
\hline $\mathrm{WC} \geq 95 \mathrm{~cm}$ & 946 & 18.7 & 35.6 & 41.7 & 50.8 & $<0.001$ \\
\hline \multicolumn{7}{|c|}{ Female } \\
\hline Overall & 3217 & 2094 & 825 & 192 & 106 & - \\
\hline High BP mm Hg & 698 & 21.0 & 22.8 & 25.1 & 20.8 & 0.467 \\
\hline $\mathrm{FBS} \geq 100 \mathrm{mg} / \mathrm{dL}$ & 1269 & 35.1 & 46.3 & 49.0 & 55.7 & $<0.001$ \\
\hline $\mathrm{TG} \geq 150 \mathrm{mg} / \mathrm{dL}$ & 1251 & 32.9 & 48.4 & 53.6 & 56.6 & $<0.001$ \\
\hline $\mathrm{HDL}<50 \mathrm{mg} / \mathrm{dL}$ & 630 & 18.5 & 20.0 & 24.0 & 30.2 & 0.009 \\
\hline $\mathrm{WC} \geq 95 \mathrm{~cm}$ & 1385 & 38.9 & 51.4 & 56.6 & 59.3 & $<0.001$ \\
\hline
\end{tabular}

Abbreviations: BP, blood pressure; FBS, fasting blood sugar; TG, triglyceride; HDL, high-density lipoprotein; WC, waist circumference

" $p$-value for trend

serum ALT in normal population. Information about the use of herbal drugs was also limited in our survey.

In conclusion, the UNL of ALT in southern Iranian women is lower than the current recommended level (i.e. > 40), while these are almost the same for men. MS components are highly common in southern Iran and are associated with elevated serum ALT. Further studies are required to estimate the UNL of the serum ALT among the Iranian population with NAFLD.

\section{ACKNOWLEDGEMENTS}

The authors would like to thank Shiraz University of Medical Sciences, Shiraz, Iran and also Center for Development of Clinical Research of Nemazee Hospital and Dr. Nasrin Shokrpour for editorial assistance.

\section{ETHICAL APPROVAL}

There is nothing to be declared.

\section{CONFLICT OF INTEREST}

The authors declare no conflict of interest related to this work.

\section{REFERENCES}

1. Pacifico L, Ferraro F, Bonci E, Anania C, Romaggioli S,
Chiesa C. Upper limit of normal for alanine aminotransferase: Quo vadis? Clin Chim Acta 2013;422:29-39. doi: 10.1016/j. cca.2013.03.030.

2. Kabir A, Pourshams A, Khoshnia M, Malekzadeh F. Normal limit for serum alanine aminotransferase level and distribution of metabolic factors in old population of Kalaleh, Iran. Hepat Mon 2013; e10640. doi: 10.5812/ hepatmon. 10640.

3. Mahady SE, Gale J, Macaskill P, Craig JC, George J. Prevalence of elevated alanine transaminase in Australia and its relationship to metabolic risk factors: A crosssectional study of 9,447 people. J Gastroenterol Hepatol 2017;32:169-76. doi: 10.1111/jgh.13434.

4. Park SH, Heo NY, Kim CH, Suk KT, Kim DJ, Lee HY. Upper reference limits for aminotransferase activities and the prevalence of elevated aminotransferase activities in a Korean population. J Clin Gastroenterol 2013;47:76-82. doi: 10.1097/MCG.0b013e31825752a4.

5. Tanaka K, Hyogo H, Ono M, Takahashi H, Kitajima Y, Ono N, et al. Upper limit of normal serum alanine aminotransferase levels in $\mathrm{J}$ apanese subjects. Hepatol Res 2014;44:1196-207. doi: 10.1111/hepr.12293.

6. Elizondo-Montemayor L, Ugalde-Casas PA, Lam-Franco L, Bustamante-Careaga H, Serrano-González M, Gutiérrez NG, et al. Association of ALT and the metabolic syndrome among Mexican children. Obes Res Clin Pract 2014;8:e79-87. doi: 10.1016/j.orcp.2012.08.191.

7. Ruhl CE, Everhart JE. Upper limits of normal for alanine aminotransferase activity in the United States population. Hepatology 2012;55:447-54. doi: 10.1002/hep.24725.

8. Hong Z, Yanfang J, Shumei H, Jie S, Qing G, Xiangwei F, et 
al. Relationship between serum aminotransferase levels and metabolic disorders in northern China. Turk J Gastroenterol 2012;23:699-707. doi:10.4318/tjg.2012.0589.

9. Wu P, Chen Q, Chen L, Zhang P, Xiao J, Chen X, et al. DoseResponse relationship between alanine aminotransferase levels within the reference interval and metabolic syndrome in chinese adults. Yonsei Med J 2017;58:158-64. doi: 10.3349/ ymj.2017.58.1.158

10. Janicko M, Veselíny E, Orencák R, Hustak R, Fedacko $\mathrm{J}$, Dražilová $\mathrm{S}$, et al. Redefining the alanine aminotransferase upper limit of normal improves the prediction of metabolic syndrome risk. Eur J Gastroenterol Hepatol 2015;27:405-11. doi: 10.1097/MEG.0000000000000297.

11. Kunde SS, Lazenby AJ, Clements RH, Abrams GA. Spectrum of NAFLD and diagnostic implications of the proposed new normal range for serum ALT in obese women. Hepatology 2005;42:650-6. doi: 10.1002/hep.20818.

12. Jamali R, Pourshams A, Amini S, Deyhim M-R, Rezvan $\mathrm{H}$, Malekzadeh R. The upper normal limit of serum alanine aminotransferase in Golestan Province, northeast Iran. Arch Iran Med 2008;11:602-7.

13. Pourshams A, Malekzadeh R, Monavvari A, Akbari MR, Mohamadkhani A, Yarahmadi S, et al. Prevalence and etiology of persistently elevated alanine aminotransferase levels in healthy Iranian blood donors. J Gastroenterol Hepatol 2005;20:229-33. doi:10.1111/j.1440-1746.2004.03511.x

14. Gandomkar A, Poustchi H, Moini M, Moghadami M, Imanieh H, Fattahi MR, et al. Pars cohort study of non-communicable diseases in Iran: protocol and preliminary results. Int J Public Health 2017;62:397-406. doi: 10.1007/s00038016-0848-2.

15. Haque T, Sasatomi E, Hayashi PH. Drug-induced liver injury: pattern recognition and future directions. Gut Liver 2016;10:27-36. doi: 10.5009/gnl15114.

16. Chen M, Suzuki A, Borlak J, Andrade RJ, Lucena MI. Drug-induced liver injury: Interactions between drug properties and host factors. J Hepatol 2015;63:503-14. doi: 10.1016/j.jhep.2015.04.016.

17. Azizi F, Hadaegh F, Khalili D, Esteghamati A, Hosseinpanah F, Delavari A, et al. Appropriate definition of metabolic syndrome among Iranian adults: report of the Iranian National Committee of Obesity. Arch Iran Med 2010;13:426-8. doi: 010135/AIM.0011.

18. Kang HS, Um SH, Seo YS, An H, Lee KG, Hyun JJ, et al. Healthy range for serum ALT and the clinical significance of "unhealthy" normal ALT levels in the Korean population. J Gastroenterol Hepatol 2011;26:292-9. doi: 10.1111/j.1440-1746.2010.06481.x.

19. Ballestri S, Zona S, Targher G, Romagnoli D, Baldelli E, Nascimbeni F, et al. Nonalcoholic fatty liver disease is associated with an almost twofold increased risk of incident type 2 diabetes and metabolic syndrome. Evidence from a systematic review and meta-analysis. $J$ Gastroenterol Hepatol 2016;31:936-44. doi: 10.1111/jgh.13264.

20. Paschos P, Paletas K. Non alcoholic fatty liver disease and metabolic syndrome. Hippokratia 2009;13:9-19.
21. Lonardo A, Ballestri S, Marchesini G, Angulo P, Loria P. Nonalcoholic fatty liver disease: a precursor of the metabolic syndrome. Dig Liver Dis 2015;47:181-90. doi: 10.1016/j.dld.2014.09.020.

22. Smart N, King N, McFarlane J, Graham P, Dieberg G. Effect of exercise training on liver function in adults who are overweight or exhibit fatty liver disease: a systematic review and meta-analysis. Br J Sports Med 2018;52:83443. doi: 10.1136/bjsports-2016-096197.

23. Khaoshbaten M, Gholami N, Sokhtehzari S, Monazami AH, Nejad MR. The effect of an aerobic exercise on serum level of liver enzymes and liver echogenicity in patients with non alcoholic fatty liver disease. Gastroenterol Hepatol Bed Bench 2013;6:S112-6.

24. Strasser B. Physical activity in obesity and metabolic syndrome. Ann N Y Acad Sci 2013;1281:141-59. doi: 10.1111/j.1749-6632.2012.06785.x.

25. Thoma C, Day CP, Trenell MI. Lifestyle interventions for the treatment of non-alcoholic fatty liver disease in adults: a systematic review. J Hepatol 2012;56:255-66. doi: 10.1016/j.jhep.2011.06.010.

26. Bae JC, Suh S, Park SE, Rhee EJ, Park CY, Oh KW, et al. Regular exercise is associated with a reduction in the risk of NAFLD and decreased liver enzymes in individuals with NAFLD independent of obesity in Korean adults. PloS One 2012;7:e46819. doi: 10.1371/journal.pone.0046819.

27. Dong MH, Bettencourt R, Barrett-Connor E, Loomba R. Alanine aminotransferase decreases with age: the Rancho Bernardo Study. PloS One 2010;5:e14254. doi: 10.1371/ journal.pone.0014254.

28. Gagliano N, Grizzi F, Annoni G. Mechanisms of aging and liver functions. Dig Dis 2007;25:118-23. doi:10.1159/000099475. 